\title{
Identification of an obesity index for predicting metabolic syndrome by gender: the rural Chinese cohort study
}

\author{
Leilei Liu', Yu Liu², Xizhuo Sun², Zhaoxia Yin², Honghui Li ${ }^{2}$ Kunpeng Deng ${ }^{3}$, Xu Chen ${ }^{1}$, Cheng Cheng ${ }^{1}$, \\ Xinping Luo ${ }^{4}$, Ming Zhang ${ }^{4}$, Linlin Li ${ }^{1}$, Lu Zhang ${ }^{1}$, Bingyuan Wang ${ }^{1,4}$, Yongcheng Ren ${ }^{1,4}$, Yang Zhao ${ }^{1,4}$,

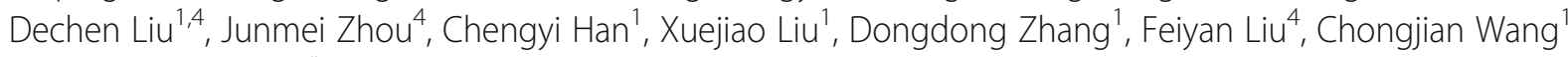 \\ and Dongsheng $\mathrm{Hu}^{1 *}$
}

\begin{abstract}
Background: To compare the accuracy of different obesity indexes, including waist circumference (WC), weightto-height ratio (WHtR), body mass index (BMI), and lipid accumulation product (LAP), in predicting metabolic syndrome (MetS) and to estimate the optimal cutoffs of these indexes in a rural Chinese adult population.

Methods: This prospective cohort involved 8468 participants who were followed up for 6 years. MetS was defined by the International Diabetes Federation, American Heart Association, and National Heart, Lung, and Blood Institute criteria. The power of the 4 indexes for predicting MetS was estimated by receiver operating characteristic (ROC) curve analysis and optimal cutoffs were determined by the maximum of Youden's index.

Results: As compared with WHtR, BMI, and LAP, WC had the largest area under the ROC curve (AUC) for predicting MetS after adjusting for age, smoking, drinking, physical activity, and education level. The AUCs (95\% Cls) for WC, WHtR, $\mathrm{BMI}$, and LAP for men and women were 0.862 (0.851-0.873) and 0.806 (0.794-0.817), $0.832(0.820-0.843)$ and 0.789 (0.777-0.801), 0.824 (0.812-0.835) and 0.790 (0.778-0.802), and 0.798 (0.785-0.810) and 0.771 (0.759-0.784), respectively. The optimal cutoffs of WC for men and women were 83.30 and $76.80 \mathrm{~cm}$. Those of WHtR, BMI, and LAP were approximately 0.51 and $0.50,23.90$ and $23.00 \mathrm{~kg} / \mathrm{m}^{2}$, and 19.23 and $20.48 \mathrm{~cm} \cdot \mathrm{mmol} / \mathrm{L}$, respectively.

Conclusions: WC as a preferred index over WHtR, BMl, and LAP for predicting MetS in rural Chinese adults of both genders; the optimal cutoffs for men and women were 83.30 and $76.80 \mathrm{~cm}$.
\end{abstract}

Keywords: Obesity index, Predict, Metabolic syndrome, Cohort study

\section{Background}

Metabolic syndrome (MetS) [1] is a cluster of metabolic abnormalities highly associated with type 2 diabetes mellitus [2], cardiovascular disease [3, 4], and all-cause mortality [5]. It is also a major and escalating public health and clinical challenge worldwide [6]. The increasing prevalence of MetS is observed all over the world and in China [7-10]. Therefore, the early prediction of MetS is essential to prevent potential severe-cardiometabolic consequences caused by MetS.

\footnotetext{
* Correspondence: hud@szu.edu.cn

'Department of Epidemiology and Health Statistics, College of Public Health, Zhengzhou University, Zhengzhou, Henan, People's Republic of China Full list of author information is available at the end of the article
}

Obesity seems to be an underlying risk factor in the development of MetS $[11,12]$. Waist circumference (WC) is used as a measure of abdominal obesity, and previous studies suggested that WC could be a powerful tool for predicting MetS $[13,14]$. However, other obesity indexes such as weight-to-height ratio (WHtR), body mass index (BMI), and lipid accumulation product (LAP) have been found better predictors of MetS than WC [15-17]. As well, controversy remains as to the superiority and the optimal cutoffs of WC, WHtR, BMI, and LAP for predicting MetS $[18,19]$.

Many previous studies had a cross-sectional design and sample sizes were small, especially studies in China [20-22]. We used data from a large prospective cohort study to

(c) The Author(s). 2018 Open Access This article is distributed under the terms of the Creative Commons Attribution 4.0 International License (http://creativecommons.org/licenses/by/4.0/), which permits unrestricted use, distribution, and reproduction in any medium, provided you give appropriate credit to the original author(s) and the source, provide a link to the Creative Commons license, and indicate if changes were made. The Creative Commons Public Domain Dedication waiver (http://creativecommons.org/publicdomain/zero/1.0/) applies to the data made available in this article, unless otherwise stated. 
compare the power of WC, WHtR, BMI, and LAP to identify an index for predicting MetS in rural Chinese adults and assess the optimal cutoffs of these indexes for predicting MetS for both genders.

\section{Methods}

\section{Study design and participants}

This prospective cohort study was conducted in rural areas around Luoyang City, Henan Province, in the middle of China. A total of 20,194 participants $\geq 18$ years old were recruited by cluster sampling at baseline (July to August of 2007 and July to August of 2008), and 17,265 participants were followed up (July to August of 2013 and July to October of 2014) (response rate 85.5\%).

For this study, we excluded participants with known MetS at baseline $(n=6390)$; incomplete data on anthropometric and laboratory measurements at baseline $(n=199)$; $\mathrm{WC} \leq 65 \mathrm{~cm}$ for men and $58 \mathrm{~cm}$ for women $(n=147)$ (based on the calculation formula of LAP [23]); no follow-up examination or death during follow-up ( $n=$ 2795); and unknown MetS status at follow-up (2195). Finally, 8468 eligible participants (4085 men) without MetS at baseline were included in the present analysis to identify the baseline obesity indexes to predict the presence of MetS at follow-up.

The study was approved by the Ethics Committee of Zhengzhou University and all participants gave their written informed consent to participate before the start of the study.

\section{Definition of metabolic syndrome}

MetS at baseline and follow-up was diagnosed according to International Diabetes Federation (IDF), American Heart Association, and National Heart, Lung, and Blood Institute (AHA/NHLBI) criteria [24]. The criteria for MetS we used was the presence of 3 or more abnormal values among the following variables: WC (90 and $80 \mathrm{~cm}$ for men and women), triglycerides (TG) level (approximately $1.69 \mathrm{mmol} /$ L), high-density lipoprotein-cholesterol (HDL-C) level (approximately 1.04 and $1.30 \mathrm{mmol} / \mathrm{L}$ for men and women), systolic blood pressure (SBP; $130 \mathrm{mmHg}$ ), diastolic blood pressure (DBP; $85 \mathrm{mmHg}$ ) and fasting plasma glucose (FPG; approximately $5.56 \mathrm{mmol} / \mathrm{L}$ ).

\section{Data collection and laboratory measurement}

Demographic and anthropometric data for each participant were collected by trained investigators who used a standard questionnaire. In the present study, smokers were defined as currently smoking and/or having smoked at least 100 cigarettes during the lifetime; the others were considered non-smokers [25]. Drinking was defined as having consumed alcohol 12 or more times in the previous year. Education level was classified as high school or above and low education level. Physical activity level was classified as low, moderate, and high physical activity level by the International Physical Activity Questionnaire scoring protocol [26].

Weight and height were measured twice to the nearest $0.5 \mathrm{~kg}$ and $0.1 \mathrm{~cm}$, respectively, with participants wearing light clothing but no shoes, according to a standard protocol [27]. BMI is an index of general obesity that combines weight and height measurements and is calculated as weight in kilograms $(\mathrm{kg})$ divided by height in meters squared $\left(\mathrm{m}^{2}\right)$ [28]. WC was measured twice at the mid-point between the lowest rib and the iliac crest

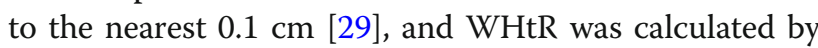
dividing WC $(\mathrm{cm})$ by height $(\mathrm{cm})$.

Blood pressure was measured by using an electronic sphygmomanometer (HEM-770AFuzzy, Omron, Japan) according to the AHA standardized protocol [30]. SBP and DBP were measured in triplicate and the results were averaged. Overnight fasting blood samples were collected for assessing levels of total cholesterol (TC), TG, HDL-C, and FPG by using an automatic biochemical analyzer (Hitachi 7080, Tokyo) with reagents from Wako Pure Chemical Industries (Osaka, Japan). Low-density lipoprotein-cholesterol (LDL-C) level was calculated by the Freidwald formula [31].

LAP was calculated by WC and TG concentration as [WC $(\mathrm{cm})-65] \times T G(\mathrm{mmol} / \mathrm{L})$ for men and $[W C(\mathrm{~cm})$ $58] \times T G(\mathrm{mmol} / \mathrm{L})$ for women, as proposed by Kahn in 2005 [23].

\section{Statistical analysis}

The baseline data for study participants are described with number (percentage) or mean (standard deviation) for categorical or quantitative variables, respectively. Participants were divided into 2 groups by presence or absence of MetS and differences between the 2 groups were examined by $\mathrm{t}$-tests for continuous variables and chi-square test for categorical variables.

Receiver operating characteristic (ROC) curves [18] were plotted to assess the performance of WC, WHtR, $\mathrm{BMI}$, and LAP in MetS prediction by gender. The model was adjusted for age and the fully adjusted model for age, smoking, drinking, physical activity, and education level. The power of MetS prediction was quantified by the area under the ROC curve (AUC) [21, 22 ] with $95 \%$ confidence intervals (CIs), a larger AUC reflecting better predictive accuracy, and $p$-values for BMI, WHtR, and LAP were computed with WC as the reference measurement. The appropriate cutoffs of the indexes were determined by the maximum of Youden's index (sensitivity + specificity -1 , with the highest sensitivity and specificity combination).

All statistical analyses involved use of MedCalc 10.1.6.0 (MedCalc Software, Ostend, Belgium). The difference was considered statistically significant at $p$-value $<0.05$ based on a 2 -sided probability. 


\section{Results}

The baseline characteristics of the study population by MetS status at follow-up are in Table 1. Compared to those without MetS men with MetS were younger, less of them were drinkers, and they had lower HDL-C levels. As expected they had higher BMI, LAP, SBP, DBP, TG, and FPG ( $p$-value < 0.05). Compared to those without MetS women with MetS were older. As expected the anthropometric and biochemical differences were similar to those in men.

The predictive values for WC, WHtR, BMI, and LAP for MetS for both genders are in Table 2 and Fig. 1. WC, WHtR, BMI, and LAP were all associated with MetS for both genders even after adjusting for age, smoking, drinking, physical activity, and education level. In the unadjusted model (Model 1), WC had the highest AUC value for men and women (0.858, 95\% CIs: 0.847-0.868 and 0.804, 95\% CIs: 0.792-0.816). On age-adjusted analysis (Model 2), WC was the most accurate for both men and women (0.862, 95\% CIs: $0.851-0.873$ and 0.805 , 95\% CIs: $0.793-0.817)$ and had the highest accuracy in the fully adjusted model (Model 3) (0.862, 95\% CIs: 0.851-0.873 and 0.806, 95\% CIs: 0.794-0.817). The AUC values for WC, WHtR, BMI, and LAP were all significantly higher for men than women in the unadjusted or adjusted model. According to the results, WC possessed the best power for predicting MetS versus the other 3 indexes on unadjusted and adjusted analyses, with no significant differences between men and women by AUC value.

Table 3 shows the gender-specific optimal cutoffs of WC, WHtR, BMI, and LAP for predicting MetS. The Youden's index indicated that the appropriate cutoffs of WC for predicting MetS for men and women were $83.30 \mathrm{~cm}$ (sensitivity $=81.34 \%$, specificity $=75.62$ and Youden's index $=0.5696$ ) and $76.80 \mathrm{~cm}$ (sensitivity $=74.01 \%$, specificity $=72.81 \%$ and Youden's index $=0.4682$ ). The Youden's index values were highest for WC for predicting MetS for both genders. Additionally, the optimal cutoffs of WHtR, BMI, and LAP for men and women were approximately 0.51 and $0.50,23.90$ and $23.00 \mathrm{~kg} / \mathrm{m}^{2}$, and 19.23 and $20.48 \mathrm{~cm} . \mathrm{mmol} / \mathrm{L}$, respectively. The present results suggested that optimal cutoffs were higher for men than women for WC, lower for LAP and similar for WHtR and BMI.

\section{Discussion}

The present study suggested that WC had the highest accuracy and appropriate cutoffs for predicting MetS for both genders as compared with other obesity indexes such as WHtR, BMI, and LAP even after adjusting for age, smoking, drinking, physical activity, and education level.

In previous studies, the obesity index with the most power for predicting MetS has been widely debated. Among some cross-sectional studies, BMI, WC, and WHtR could similarly predict the presence of multiple metabolic risk factors in Chinese people [32]. However,
WHtR was a better index for screening MetS based on the IDF criteria as compared with BMI and LAP for both genders in a Xinjiang population [22]. In a population-based study in China [20], WHtR was the best predictor of MetS in men, but WHtR and WC were equally good predictors of MetS in women. In addition, LAP was a powerful tool for predicting MetS in undiagnosed Brazilian adults [17]. The HANDLS study [14] suggested that WC was the most powerful tool for predicting MetS among adults. Likewise, WC had the highest AUC value as compared with WHtR and LAP in a study of older men and women [33]. Additionally, in a study of adults in northeast China [34], WC was superior to BMI and WHtR in predicting MetS in men, but WHtR was superior to BMI and WC in predicting MetS in women. Among some cohort designs, the San Antonio Heart Study suggested that BMI and WC had equal power in predicting MetS in non-Hispanic whites and Mexican Americans [11]. In contrast, in an Iranian population in the north of Iran, LAP had strong and reliable diagnostic accuracy in predicting MetS, with better predictability than WC, WHtR, and BMI [35]. The Korean Genome and Epidemiology Study [36] suggested that WHtR was a better discriminator of MetS than WC and BMI. The most appropriate index and the accuracy for predicting MetS may depend on ethnicity, age, gender or the diagnostic criteria of MetS, given these inconsistent results.

The present study suggested that the cutoffs of WC for predicting MetS in rural Chinese men and women were 83.30 and $76.80 \mathrm{~cm}$. A population-based study of Chinese people suggested that the optimal cutoffs of WC for men and women were 84.8 and $75.8 \mathrm{~cm}$ [21], which is similar to our findings, but lower than the IDF-suggested cutoffs for Chinese men and women of 90 and $80 \mathrm{~cm}$ [37]. As well, 2 population-based surveys conducted in China [32, 38] suggested higher WC cutoffs than those in our study. In addition, data from a cross-sectional study of 203 older Brazilians showed WC cutoffs of $90.90 \mathrm{~cm}$ for men and $80.20 \mathrm{~cm}$ for women [33]. The different ethnicities and race, cross-sectional design, and small sample size may lead to inconsistent results. Further study is needed to explore the application of current WC cutoffs to healthy people in the real world. WHtR cutoffs were 0.51 and 0.53 for Chinese men and women, respectively [32], and our results were the same as these findings and from other studies [34, 38]. The appropriate cutoffs of BMI we found among rural Chinese men and women were approximately 23.90 and $23.00 \mathrm{~kg} / \mathrm{m}^{2}$, which agreed with findings from population-based studies of Chinese adults [21, 32]. However, the cutoffs of LAP were lower than in previous research. The optimal cutoffs of LAP were previously found to be 34.7 and $27.3 \mathrm{~cm} . \mathrm{mmol} / \mathrm{L}$ for Chinese men and women, respectively [19], and 24.76 and $26.49 \mathrm{~cm} . \mathrm{mmol} / \mathrm{L}$ 
Table 1 Baseline characteristics of study participants by metabolic syndrome (MetS) status at follow-up

\begin{tabular}{|c|c|c|c|c|}
\hline Variables & Total & With MetS & Without MetS & $p$-value \\
\hline & $(n=8468)$ & $(n=1825)$ & $3576(8754)$ & \\
\hline Men & $4085(48.24)$ & $509(12.46)$ & $3576(87.54)$ & \\
\hline Age (years) & $52.38(13.07)$ & $50.13(12.77)$ & $52.70(13.08)$ & $<0.001$ \\
\hline Smoking & $2866(70.16)$ & $357(70.14)$ & $2509(70.16)$ & 0.991 \\
\hline Drinking & $1063(26.02)$ & $173(33.99)$ & $890(24.89)$ & $<0.001$ \\
\hline Education level & & & & 0.228 \\
\hline High school or above & $617(15.10)$ & $86(16.90)$ & $531(14.85)$ & \\
\hline Physical activity & & & & 0.079 \\
\hline Low & $931(22.79)$ & $125(24.56)$ & $806(22.54)$ & \\
\hline Moderate & $724(17.72)$ & $103(20.24)$ & $621(17.37)$ & \\
\hline High & $2430(59.49)$ & $281(55.21)$ & $2149(60.10)$ & \\
\hline WC (cm) & $79.59(7.45)$ & $88.33(6.66)$ & $78.34(6.68)$ & $<0.001$ \\
\hline WHtR & $0.48(0.05)$ & $0.53(0.04)$ & $0.48(0.04)$ & $<0.001$ \\
\hline BMI $\left(\mathrm{kg} / \mathrm{m}^{2}\right)$ & $22.55(2.49)$ & $25.17(2.46)$ & $22.17(2.26)$ & $<0.001$ \\
\hline LAP (cm.mmol/L) & $21.23(17.02)$ & $37.11(20.08)$ & $18.97(15.25)$ & $<0.001$ \\
\hline TC $(\mathrm{mmol} / \mathrm{L})$ & $4.29(0.85)$ & $4.43(0.86)$ & $4.28(0.85)$ & $<0.001$ \\
\hline TG (mmol/L) & $1.37(0.66)$ & $1.60(0.75)$ & $1.34(0.65)$ & $<0.001$ \\
\hline $\mathrm{SBP}(\mathrm{mmHg})$ & $123.84(17.88)$ & $127.82(19.14)$ & $123.28(17.62)$ & $<0.001$ \\
\hline $\mathrm{DBP}(\mathrm{mmHg})$ & $76.65(10.69)$ & $80.12(11.13)$ & $76.16(10.54)$ & $<0.001$ \\
\hline FPG (mmol/L) & $5.44(1.24)$ & $5.59(1.41)$ & $5.42(1.21)$ & 0.009 \\
\hline $\mathrm{HDL}-\mathrm{C}(\mathrm{mmol} / \mathrm{L})$ & $1.14(0.25)$ & $1.08(0.22)$ & $1.15(0.25)$ & $<0.001$ \\
\hline LDL-C (mmol/L) & $2.53(0.72)$ & $2.62(0.72)$ & $2.52(0.71)$ & 0.004 \\
\hline Women & $4383(51.76)$ & $1316(30.03)$ & $3067(69.97)$ & \\
\hline Age (years) & $47.92(12.45)$ & $49.50(11.23)$ & $47.25(12.88)$ & $<0.001$ \\
\hline Smoking & $13(0.30)$ & $4(0.30)$ & $9(0.29)$ & 0.953 \\
\hline Drinking & $31(0.71)$ & $8(0.61)$ & $23(0.75)$ & 0.607 \\
\hline Education level & & & & 0.079 \\
\hline High school or above & $337(7.69)$ & $87(6.61)$ & $250(8.15)$ & \\
\hline Physical activity & & & & 0.065 \\
\hline Low & $1358(30.98)$ & $381(28.95)$ & 977 (31.86) & \\
\hline Moderate & $1035(23.61)$ & $316(24.01)$ & 719 (23.44) & \\
\hline High & $1990(45.40)$ & $619(47.04)$ & $1371(44.70)$ & \\
\hline$W C(\mathrm{~cm})$ & $75.72(7.85)$ & $81.53(7.86)$ & $73.22(6.39)$ & $<0.001$ \\
\hline WHtR & $0.49(0.05)$ & $0.53(0.05)$ & $0.48(0.04)$ & $<0.001$ \\
\hline BMI $\left(\mathrm{kg} / \mathrm{m}^{2}\right)$ & $22.75(2.93)$ & $24.75(2.98)$ & $21.89(2.45)$ & $<0.001$ \\
\hline LAP (cm.mmol/L) & $23.10(15.52)$ & $32.73(16.85)$ & $18.98(12.88)$ & $<0.001$ \\
\hline TC (mmol/L) & $4.40(0.89)$ & $4.56(0.90)$ & $4.33(0.88)$ & $<0.001$ \\
\hline TG $(\mathrm{mmol} / \mathrm{L})$ & $1.28(0.62)$ & $1.42(0.65)$ & $1.22(0.59)$ & $<0.001$ \\
\hline $\mathrm{SBP}(\mathrm{mmHg})$ & $120.47(19.45)$ & $125.18(19.53)$ & 118.46 (19.06) & $<0.001$ \\
\hline $\mathrm{DBP}(\mathrm{mmHg})$ & $75.73(10.59)$ & $79.15(10.90)$ & $74.27(10.10)$ & $<0.001$ \\
\hline FPG (mmol/L) & $5.37(1.11)$ & $5.48(1.26)$ & $5.33(1.04)$ & $<0.001$ \\
\hline $\mathrm{HDL}-\mathrm{C}(\mathrm{mmol} / \mathrm{L})$ & $1.27(0.27)$ & $1.23(0.26)$ & $1.29(0.28)$ & $<0.001$ \\
\hline LDL-C (mmol/L) & $2.54(0.74)$ & $2.68(0.75)$ & $2.49(0.73)$ & $<0.001$ \\
\hline
\end{tabular}

Abbreviations: WC waist circumference, WHtR waist-to-height ratio, BMI body mass index, LAP lipid accumulation product, TC total cholesterol, TG triglycerides, $S B P$ systolic blood pressure, $D B P$ diastolic blood pressure, FPG fasting plasma glucose, $H D L-C$ high-density lipoprotein-cholesterol, $L D L-C$ low-density lipoprotein-cholesterol

Data are number (percentage) or mean (standard deviation) 
Table 2 AUC values for WC, WHtR, BMI, and LAP for predicting MetS by gender

\begin{tabular}{|c|c|c|c|c|c|}
\hline \multirow[t]{2}{*}{ Model } & \multirow[t]{2}{*}{ Variables } & \multicolumn{2}{|l|}{ Men $(n=4085)$} & \multicolumn{2}{|l|}{ Women $(n=4383)$} \\
\hline & & AUC (95\% Cls) & $p$-value & AUC (95\% Cls) & $p$-value \\
\hline \multirow[t]{4}{*}{ Model 1} & WC & $0.858(0.847-0.868)$ & - & $0.804(0.792-0.816)$ & - \\
\hline & WHtR & $0.819(0.807-0.831)$ & $<0.001$ & $0.789(0.776-0.801)$ & $<0.001$ \\
\hline & $\mathrm{BMl}$ & $0.821(0.808-0.832)$ & $<0.001$ & $0.781(0.768-0.793)$ & $<0.001$ \\
\hline & LAP & $0.796(0.783-0.808)$ & $<0.001$ & $0.770(0.758-0.783)$ & $<0.001$ \\
\hline \multirow[t]{4}{*}{ Model 2} & WC & $0.862(0.851-0.873)$ & - & $0.805(0.793-0.817)$ & - \\
\hline & WHtR & $0.831(0.820-0.843)$ & $<0.001$ & $0.789(0.776-0.801)$ & $<0.001$ \\
\hline & $\mathrm{BMl}$ & $0.823(0.811-0.835)$ & $<0.001$ & $0.790(0.778-0.802)$ & 0.012 \\
\hline & LAP & $0.798(0.785-0.810)$ & $<0.001$ & $0.770(0.758-0.783)$ & $<0.001$ \\
\hline \multirow[t]{4}{*}{ Model 3} & WC & $0.862(0.851-0.873)$ & - & $0.806(0.794-0.817)$ & - \\
\hline & WHtR & $0.832(0.820-0.843)$ & $<0.001$ & $0.789(0.777-0.801)$ & $<0.001$ \\
\hline & $\mathrm{BMl}$ & $0.824(0.812-0.835)$ & $<0.001$ & $0.790(0.778-0.802)$ & 0.010 \\
\hline & LAP & $0.798(0.785-0.810)$ & $<0.001$ & $0.771(0.759-0.784)$ & $<0.001$ \\
\hline
\end{tabular}

Abbreviations: AUC the area under the ROC curve, WC waist circumference, WHtR waist-to-height ratio, BMI body mass index, LAP lipid accumulation product Model 1: unadjusted model

Model 2: adjusted for age

Model 3: adjusted for age, smoking, drinking, physical activity, and education level

${ }^{*} p$-value indicates the statistical significance of other models compared with a model of WC

in the Kazakh adult population in Xinjiang [22]. Additionally, the HANDLS study [14] suggested that optimal cutoffs of various indexes may differ by gender, and we found this trend for WC and LAP but not WHtR and BMI.

The determinant (WC or WC plus TG) is a component of MetS, but in terms of considering the determinant as a potential confounder, we could not consider MetS as a cluster of metabolic abnormalities highly associated with type 2 diabetes mellitus and cardiovascular disease, which occurs together half the time than accidentally alone [24]. As well, although one study showed that confounding occurs in evaluating classification accuracy when a variable is associated with both the marker and the binary outcome [39], many previous studies showed that only age and gender were potential confounders of the association of WC or LAP with MetS [17, 23, 35, 40-42]. We conducted the gender-specific study and adjusted for age in the present study, so the results do not have a bias of accuracy.

The primary purpose of this study was to define the baseline characteristics predicting the presence of MetS at follow-up based on a rural Chinese population. The sample size of this study was not determined specifically; 20,194 cohort members were recruited at baseline examination and followed up for 6 years currently. Therefore,

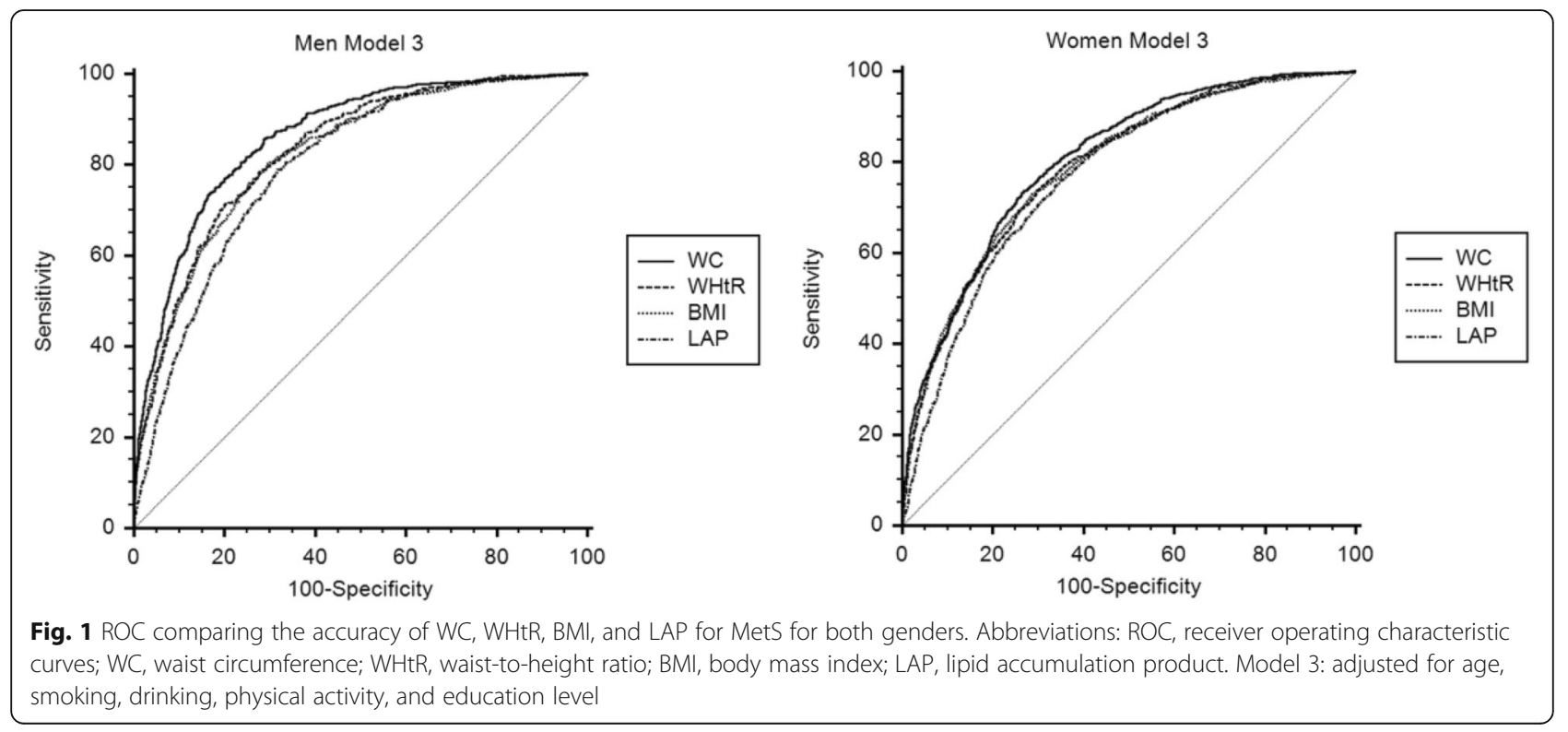


Table 3 Optimal cutoffs of WC, WHtR, BMI, and LAP for MetS prediction by gender

\begin{tabular}{lllll}
\hline Variables & Cutoff & $\begin{array}{l}\text { Sensitivity } \\
(\%)\end{array}$ & $\begin{array}{l}\text { Specificity } \\
(\%)\end{array}$ & $\begin{array}{l}\text { Youden's } \\
\text { index }\end{array}$ \\
\hline Men & & & & \\
WC (cm) & 83.30 & 81.34 & 75.62 & 0.5696 \\
WHtR & 0.51 & 73.08 & 76.43 & 0.4951 \\
BMI (kg/m²) & 23.90 & 72.69 & 76.99 & 0.4968 \\
LAP (cm.mmol/ & 19.23 & 84.28 & 61.94 & 0.4622 \\
L) & & & & \\
Women & & & & \\
WC (cm) & 76.80 & 74.01 & 72.81 & 0.4682 \\
WHtR & 0.50 & 74.39 & 69.61 & 0.4400 \\
BMI (kg/m $\left.{ }^{2}\right)$ & 23.00 & 71.28 & 70.33 & 0.4161 \\
LAP (cm.mmol/L) & 20.48 & 76.44 & 64.62 & 0.4106 \\
\hline
\end{tabular}

Abbreviations: WC waist circumference, WHtR waist-to-height ratio, BMI body mass index, LAP lipid accumulation product

the sample size should be large enough to meet most of the study hypotheses for MetS conditions.

Many previous studies had limitations that included a cross-sectional design [32-34], but our study's strength lay in its prospective design. Furthermore, we used data from a large population-based-cohort of both genders as compared with previous studies with a small sample size [11, 19-21]. Additionally, we compared various indexes for predicting MetS among rural Chinese adults and provided corresponding AUC and cutoffs for these indexes in this analysis stratified by gender.

We are aware of several relevant limitations of our study besides the strengths we have mentioned. First, although we adjusted for age, smoking, drinking, physical activity, and education level, other confounders might have affected MetS, such as family history of disease, which were not included in the adjustment model. Second, participants were exclusively from rural areas in China, so our results may not be transferred to urban populations. Finally, 2929 participants were not followed up in this cohort, which could imply follow-up bias.

In conclusion, we provide longitudinal evidence for the power of WC, WHtR, BMI, and LAP in predicting MetS, and all 4 indexes were significantly associated with MetS for both genders even after adjusting for some known confounding variables. In addition, WC showed superior power for predicting MetS as compared with the other 3 indexes. The use of a simple index such as WC could contribute to the early prediction of MetS in rural Chinese people, as effective intervention to prevent and treat risks related to MetS. In addition, it may provide useful instruction for public health promotion to maintain optimal cutoffs of WC, BMI, WHtR, and LAP.

Nevertheless, from our results and those of previous research, controversy still remains as to which index has better accuracy for predicting MetS in different countries, ethnicities, and genders. Thus, further larger and prospective research is warranted to elucidate the association between the 4 obesity indexes and MetS and to define appropriate cutoffs in the adult Chinese population.

The WC cutoffs of $83.30 \mathrm{~cm}$ for men and $76.80 \mathrm{~cm}$ for women from our study are quite a lot different from the $90 \mathrm{~cm}$ for Asian American men and $80 \mathrm{~cm}$ for Asian American women used in the IDF and AHA/NHLBI criteria [24]. In Japan specific WC cutoffs of $85 \mathrm{~cm}$ for men and $90 \mathrm{~cm}$ for women were based on visceral fat quantitation on CT scan [43, 44]. Thus country and ethnic specific criteria based on good local data are an appropriate approach. If the cutoffs derived from our data are to be used in practice, we may need to use $83 \mathrm{~cm}$ or even $85 \mathrm{~cm}$ for men and $77 \mathrm{~cm}$ or even $75 \mathrm{~cm}$ for women for simplicity but these would still be different from those used for Asian American participants.

\section{Conclusions}

In summary, the present prospective cohort study found that WC at cutoffs $83.30 \mathrm{~cm}$ for men and $76.80 \mathrm{~cm}$ for women was superior to BMI, WHtR, and LAP for predicting MetS in rural Chinese adults. It is crucial for the early prediction and prevention of MetS to indentify an appropriate index and corresponding optimal cutoffs.

\section{Abbreviations \\ AUC: the area under the ROC curve; BMI: body mass index; Cls: confidence intervals; DBP: diastolic blood pressure; FPG: fasting plasma glucose; HDL- C: high-density lipoprotein-cholesterol; LAP: lipid accumulation product; LDL- C: low-density lipoprotein-cholesterol; MetS: metabolic syndrome; \\ ROC: receiver operating characteristic; SBP: systolic blood pressure; TC: total cholesterol; TG: triglycerides; WC: waist circumference; WHtR: weight-to- height ratio}

\section{Acknowledgments}

We are indebted to all the subjects participating in this study.

\section{Funding}

This study was supported by the National Natural Science Foundation of China (No. 81373074, 81402752, and 81673260) and Science and Technology Development Foundation of Shenzhen (No. JCYJ20160307155707264).

\section{Availability of data and materials}

The study was initially designed for data not to be publicly available, but data will now be provided upon approved request.

\section{Authors' contributions}

LL1, LL2, MZ, CW, and DH. substantially contributed to the design and drafting of the study and the analysis and interpretation of the data. $L L 1, Y L, X S, Z Y, H L$, and $K D$. contributed to the data analysis and discussion. $X C, C C, X L 2, L Z, B W, Y R, Y Z$, and $D L$. made contributions to acquisition and interpretation of data. $\mathrm{JZ}, \mathrm{CH}, \mathrm{XL1}, \mathrm{DZ}, \mathrm{FL}$, and $\mathrm{DH}$. revised it critically for important intellectual content. All authors read and approved the final manuscript.

Ethics approval and consent to participate

The study was approved by the Ethics Committee of Zhengzhou University and all participants gave their written informed consent to participate before the start of the study. 


\section{Consent for publication}

Not applicable.

\section{Competing interests}

The authors declare that they have no competing interests.

\section{Publisher's Note}

Springer Nature remains neutral with regard to jurisdictional claims in published maps and institutional affiliations.

\section{Author details}

'Department of Epidemiology and Health Statistics, College of Public Health, Zhengzhou University, Zhengzhou, Henan, People's Republic of China. ${ }^{2}$ The Affiliated Luohu Hospital of Shenzhen University Health Sciences Center, Shenzhen, Guangdong, People's Republic of China. ${ }^{3}$ Yantian Entry-exit Inspection and Quarantine Bureau, Shenzhen, Guangdong, People's Republic of China. ${ }^{4}$ Department of Preventive Medicine, Shenzhen University Health Sciences Center, Shenzhen, Guangdong, People's Republic of China.

\section{Received: 18 October 2017 Accepted: 24 July 2018}

\section{Published online: 06 August 2018}

\section{References}

1. Eckel RH, Grundy SM, Zimmet PZ. The metabolic syndrome. Lancet. 2005; 365(9468):1415-28.

2. Grundy SM. Metabolic syndrome pandemic. Arterioscler Thromb Vasc Biol. 2008;28(4):629-36.

3. Grundy SM. Metabolic syndrome: connecting and reconciling cardiovascular and diabetes worlds. J Am Coll Cardiol. 2006;47(6):1093-100.

4. Mottillo S, Filion KB, Genest J, Joseph L, Pilote L, Poirier P, Rinfret S, Schiffrin $E L$, Eisenberg MJ. The metabolic syndrome and cardiovascular risk a systematic review and meta-analysis. J Am Coll Cardiol. 2010;56(14):1113-32.

5. Wu SH, Liu Z, Ho SC. Metabolic syndrome and all-cause mortality: a metaanalysis of prospective cohort studies. Eur J Epidemiol. 2010;25(6):375-84.

6. O'Neill S, O'Driscoll L. Metabolic syndrome: a closer look at the growing epidemic and its associated pathologies. Obesity reviews : an official journal of the International Association for the Study of Obesity. 2015;16(1):1-12.

7. Cai H, Huang J, Xu G, Yang Z, Liu M, Mi Y, Liu W, Wang H, Qian D. Prevalence and determinants of metabolic syndrome among women in Chinese rural areas. PLoS One. 2012;7(5):e36936.

8. Song P, Yu J, Chang X, Wang M, An L: Prevalence and Correlates of Metabolic Syndrome in Chinese Children: The China Health and Nutrition Survey. Nutrients 2017, 9(1).

9. Mozumdar A, Liguori G. Persistent increase of prevalence of metabolic syndrome among U.S. adults: NHANES III to NHANES. Diabetes care 2011. 1999-2006;34(1):216-9.

10. Xi B, He D, Hu Y, Zhou D. Prevalence of metabolic syndrome and its influencing factors among the Chinese adults: the China health and nutrition survey in 2009. Prev Med. 2013;57(6):867-71.

11. Han TS, Williams K, Sattar N, Hunt KJ, Lean MEJ, Haffner SM. Analysis of obesity and Hyperinsulinemia in the development of metabolic syndrome: San Antonio heart study. Obes Res. 2002;10(9):923-31.

12. Yong-Woo Park M. PhD, Shankuan Zhu, MD, PhD, Latha Palaniappan, MD, Stanley, Heshka, PhD, Mercedes R. Carnethon, PhD, and Steven B. Heymsfield, MD: the metabolic syndrome: prevalence and associated risk factor findings in the US population from the third National Health and nutrition examination survey, 1988-1994. Arch Intern Med. 2003;24: 427-36.

13. Agredo-Zuniga RA, Aguilar-de Plata C, Suarez-Ortegon MF. Waist:height ratio, waist circumference and metabolic syndrome abnormalities in Colombian schooled adolescents: a multivariate analysis considering located adiposity. Br J Nutr. 2015;114(5):700-5.

14. Beydoun MA, Kuczmarski MT, Wang Y, Mason MA, Evans MK, Zonderman AB. Receiver-operating characteristics of adiposity for metabolic syndrome: the healthy aging in neighborhoods of diversity across the life span (HANDLS) study. Public Health Nutr. 2011;14(1): 77-92.

15. Sakurai M, Takamura T, Miura K, Kaneko S, Nakagawa H. BMI may be better than waist circumference for defining metabolic syndrome in Japanese women. Diabetes Care. 2008;31(3):e12.
16. Rodea-Montero ER, Evia-Viscarra ML, Apolinar-Jimenez E. Waist-to-height ratio is a better anthropometric index than waist circumference and BMI in predicting metabolic syndrome among obese Mexican adolescents. Int J Endocrinol. 2014;2014:195407.

17. Nascimento-Ferreira MV, Rendo-Urteaga T, Vilanova-Campelo RC, Carvalho HB, da Paz OG, Paes Landim MB, Torres-Leal FL. The lipid accumulation product is a powerful tool to predict metabolic syndrome in undiagnosed Brazilian adults. Clin Nutr. 2017;36(6):1693-700.

18. Yang F, LV JH, Lei SF, Chen XD, Liu MY, Jian WX, Xu H, Tan LJ, Deng FY, Yang $Y$ J, et al. Receiver-operating characteristic analyses of body mass index, waist circumference and waist-to-hip ratio for obesity: screening in young adults in central south of China. Clin Nutr. 2006;25(6):1030-9.

19. Guo SX, Zhang XH, Zhang JY, He J, Yan YZ, Ma JL, Ma RL, Guo H, Mu LT, Li $S G$, et al. Visceral adiposity and anthropometric indicators as screening tools of metabolic syndrome among low income rural adults in Xinjiang. Sci Rep. 2016;6:36091.

20. Zhang ZQ, Deng J, He LP, Ling WH, Su YX, Chen YM. Comparison of various anthropometric and body fat indices in identifying cardiometabolic disturbances in Chinese men and women. PLoS One. 2013;8(8):e70893.

21. Zeng Q, He Y, Dong S, Zhao X, Chen Z, Song Z, Chang G, Yang F, Wang Y. Optimal cut-off values of BMl, waist circumference and waist:height ratio for defining obesity in Chinese adults. Br J Nutr. 2014;112(10):1735-44.

22. Zhang XH, Zhang M, He J, Yan YZ, Ma JL, Wang K, Ma RL, Guo H, Mu LT, Ding YS, et al. Comparison of anthropometric and Atherogenic indices as screening tools of metabolic syndrome in the Kazakh adult population in Xinjiang. Int J Environ Res Public Health. 2016;13(4):428.

23. Kahn HS. The "lipid accumulation product" performs better than the body mass index for recognizing cardiovascular risk: a population-based comparison. BMC Cardiovasc Disord. 2005;5:26.

24. Alberti KG, Eckel RH, Grundy SM, Zimmet PZ, Cleeman Jl, Donato KA, Fruchart JC, James WP, Loria CM, Smith SC Jr, et al. Harmonizing the metabolic syndrome: a joint interim statement of the international diabetes federation task force on epidemiology and prevention; National Heart, Lung, and Blood Institute; American Heart Association; world heart federation; international atherosclerosis society; and International Association for the Study of obesity. Circulation. 2009;120(16):1640-5.

25. Wildman RP, Muntner P, Reynolds K, McGinn AP, Rajpathak S, Wylie-Rosett J, Sowers MR. The obese without cardiometabolic risk factor clustering and the normal weight with cardiometabolic risk factor clustering: prevalence and correlates of 2 phenotypes among the US population (NHANES 19992004). Arch Intern Med. 2008;168(15):1617-24.

26. Craig CL, Marshall AL, Sjostrom M, Bauman AE, Booth ML, Ainsworth BE, Pratt M, Ekelund U, Yngve A, Sallis JF, et al. International physical activity questionnaire: 12-country reliability and validity. Med Sci Sports Exerc. 2003; 35(8):1381-95

27. Ulla Uusitalo AC, Liisa Palonen, Milorad Toša Zikic, et al.: Geographical variation in the major risk factors of coronary heart disease in men and women aged 35-64 years. Wld hlth statist quan 1988, 41:115-140.

28. Doak CM, Hoffman DJ, Norris SA, Campos Ponce M, Polman K, Griffiths PL. Is body mass index an appropriate proxy for body fat in children? Global Food Security. 2013:2(2):65-71.

29. Wang CJ, Li YQ, Wang L, Li LL, Guo YR, Zhang LY, Zhang MX, Bie RH. Development and evaluation of a simple and effective prediction approach for identifying those at high risk of dyslipidemia in rural adult residents. PLoS One. 2012;7(8):e43834.

30. Perloff D, Grim C, Flack J, Frohlich ED, Hill M, Mcdonald M, Morgenstern BZ. Human blood-pressure determination by Sphygmomanometry. Circulation. 1993:88(5):2460-70

31. Bairaktari E, Hatzidimou K, Tzallas C, Vini M, Katsaraki A, Tselepis A, Elisaf M, Tsolas O. Estimation of LDL cholesterol based on the Friedewald formula and on apo B levels. Clin Biochem. 2000;33(7):549-55.

32. Liu Y, Tong G, Tong W, Lu L, Qin X. Can body mass index, waist circumference, waist-hip ratio and waist-height ratio predict the presence of multiple metabolic risk factors in Chinese subjects? BMC Public Health. 2011:11:35.

33. de Oliveira CC, Roriz AK, Ramos LB, Gomes Neto M. Indicators of adiposity predictors of metabolic syndrome in the elderly. Annals of nutrition \& metabolism. 2017;70(1):9-15.

34. Yu J, Tao Y, Tao Y, Yang S, Yu Y, Li B, Jin L. Optimal cut-off of obesity indices to predict cardiovascular disease risk factors and metabolic syndrome among adults in Northeast China. BMC Public Health. 2016; 16(1):1079. 
35. Motamed N, Razmjou S, Hemmasi G, Maadi M, Zamani F. Lipid accumulation product and metabolic syndrome: a population-based study in northern Iran, Amol. J Endocrinol Investig. 2016;39(4):375-82.

36. Ko KP, Oh DK, Min H, Kim CS, Park JK, Kim Y, Kim SS. Prospective study of optimal obesity index cutoffs for predicting development of multiple metabolic risk factors: the Korean genome and epidemiology study. J Epidemiol. 2012;22(5):433-9.

37. Saito I. Epidemiological evidence of type 2 diabetes mellitus, metabolic syndrome, and cardiovascular disease in Japan. Circulation journal : official journal of the Japanese Circulation Society. 2012;76(5):1066-73.

38. Shao J, Yu L, Shen X, Li D, Wang K. Waist-to-height ratio, an optimal predictor for obesity and metabolic syndrome in Chinese adults. J Nutr Health Aging. 2010;14(9):782-5.

39. Janes H, Pepe MS. Adjusting for covariates in studies of diagnostic, screening, or prognostic markers: an old concept in a new setting. Am J Epidemiol. 2008;168(1):89-97.

40. Chiang JK, Koo M. Lipid accumulation product: a simple and accurate index for predicting metabolic syndrome in Taiwanese people aged 50 and over. BMC Cardiovasc Disord. 2012;12:78.

41. Tellechea ML, Aranguren F, Martinez-Larrad MT, Serrano-Rios M, Taverna MJ, Frechtel GD. Ability of lipid accumulation product to identify metabolic syndrome in healthy men from Buenos Aires. Diabetes Care. 2009;32(7):e85.

42. Taverna MJ, Martinez-Larrad MT, Frechtel GD, Serrano-Rios M. Lipid accumulation product: a powerful marker of metabolic syndrome in healthy population. Eur J Endocrinol. 2011;164(4):559-67.

43. Yuji M. Metabolic syndrome-definition and diagnostic criteria in Japan. J Atheroscler Thromb. 2005;12(6):301.

44. Yuji M. Metabolic syndrome-definition and diagnostic criteria in Japan. J Jpn Soc Intern Med. 2005;94:188-203.

Ready to submit your research? Choose BMC and benefit from:

- fast, convenient online submission

- thorough peer review by experienced researchers in your field

- rapid publication on acceptance

- support for research data, including large and complex data types

- gold Open Access which fosters wider collaboration and increased citations

- maximum visibility for your research: over $100 \mathrm{M}$ website views per year

At $\mathrm{BMC}$, research is always in progress.

Learn more biomedcentral.com/submissions 